OPEN ACCESS

Edited by:

Cesar Wong,

Hong Kong Polytechnic University,

Hong Kong

Reviewed by:

Fatah Kashanchi,

George Mason University,

United States

HF Tsang,

Hong Kong Adventist Hospital,

Hong Kong

${ }^{*}$ Correspondence:

Hao Zhang

haolabcancercenter@163.com

†'Shared co-first authorship

Specialty section:

This article was submitted to

RNA,

a section of the journa

Frontiers in Genetics

Received: 17 September 2018

Accepted: 26 February 2019

Published: 14 March 2019

Citation:

Yu J, Lin Y, Xiong X, Li K, Yao Z,

Dong $H$, Jiang $Z$, Yu D, Yeung S-CJ and Zhang $H$ (2019) Detection of Exosomal PD-L1 RNA in Saliva of Patients With Periodontitis.

Front. Genet. 10:202

doi: 10.3389/fgene.2019.00202

\section{Detection of Exosomal PD-L1 RNA in Saliva of Patients With Periodontitis}

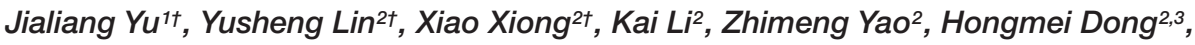 \\ Zuojie Jiang ${ }^{2}$, Dan Yu' ${ }^{1}$, Sai-Ching Jim Yeung ${ }^{4,5}$ and Hao Zhang ${ }^{3,6 *}$

\begin{abstract}
${ }^{1}$ Department of Stomatology, The First Affiliated Hospital of Shantou University Medical College, Shantou, China, ${ }^{2}$ Cancer Research Center, Shantou University Medical College, Shantou, China, ${ }^{3}$ Institute of Precision Cancer and Pathology, Jinan University Medical College, Guangzhou, China, ${ }^{4}$ Department of Emergency Medicine, University of Texas MD Anderson Cancer Center, Houston, TX, United States, ${ }^{5}$ Department of Endocrine Neoplasia and Hormonal Disorders, University of Texas MD Anderson Cancer Center, Houston, TX, United States, ${ }^{6}$ Research Center of Translational Medicine, The Second Affiliated Hospital of Shantou University Medical College, Shantou, China
\end{abstract}

Periodontitis is the most prevalent inflammatory disease of the periodontium, and is related to oral and systemic health. Exosomes are emerging as non-invasive biomarker for liquid biopsy. We here evaluated the levels of programmed death-ligand 1 (PD-L1) mRNA in salivary exosomes from patients with periodontitis and non-periodontitis controls. The purposes of this study were to establish a procedure for isolation and detection of mRNA in exosomes from saliva of periodontitis patients, to characterize the level of salivary exosomal $P D-L 1$, and to illustrate its clinical relevance. Bioinformatics analysis suggested that periodontitis was associated with an inflammation gene expression signature, that $P D-L 1$ expression positively correlated with inflammation in periodontitis based on gene set enrichment analysis (GSEA) and that $P D-L 1$ expression was remarkably elevated in periodontitis patients versus control subjects. Exosomal RNAs were successfully isolated from saliva of 61 patients and 30 controls and were subjected to qRT-PCR. Levels of $P D-L 1 \mathrm{mRNA}$ in salivary exosomes were higher in periodontitis patients than controls $(P<0.01)$. Salivary exosomal $P D-L 1 \mathrm{mRNA}$ showed significant difference between the stages of periodontitis. In summary, the protocols for isolating and detecting exosomal RNA from saliva of periodontitis patients were, for the first time, characterized. The current study suggests that assay of exosomes-based $P D-L 1$ mRNA in saliva has potential to distinguish periodontitis from the healthy, and the levels correlate with the severity/stage of periodontitis.

Keywords: immune checkpoint, exosomes, saliva, chronic periodontal disease, biomarker, disease stage

\section{INTRODUCTION}

Periodontitis is one of the most prevalent disease in dentistry, impairing the integrity of the periodontium and leading ultimately to tooth loss. Periodontitis is a chronic inflammatory disease caused by microorganisms colonizing the dentogingival interface (Slots, 2013). Currently, the diagnosis of periodontitis was mostly based on clinical and radiographic evaluations without specific evaluation of the underlying inflammatory response (Buduneli and Kinane, 2011; Baeza et al., 2016). Measuring electrolyte concentration in gingival crevicular fluid (GCF), especially the 
concentration of sodium, potassium and calcium ions, reflects the clinical status of the periodontal tissues such that the pattern of concentrations of these ions may be used as a potential diagnostic marker for active periodontitis (Koregol et al., 2011).

Given the close relevance of inflammation to periodontitis, immune-regulatory factors have been explored as biomarker of periodontitis (Han et al., 2012; Kimak et al., 2015). Programmed death-ligand 1 (PD-L1), also known as the $\mathrm{B} 7-\mathrm{H} 1$ receptor, plays an important role in cell-mediated immune responses (Gianchecchi et al., 2013). PD-L1 regulates T cell activation and tolerance, and is able to inhibit activated $\mathrm{T}$ cell functions and survival (Kim et al., 2005). High expression of PD-L1 in host cells may contribute to the chronicity of inflammatory disorders (Zamani et al., 2016).

PD-L1 is involved in periodontitis. Porphyromonas gingivalis ( $P$. gingivalis) is a keystone pathogen in chronic periodontitis (Groeger et al., 2017), and it induces expression of PD-L1 in malignant and non-malignant oral epithelial cells (Groeger et al., 2017). In periodontitis, $P$. gingivalis inhibits the synthesis of cytokines and increases humoral responses. This reduces the inflammatory responses related to $\mathrm{T}$ - and B-cell activation, and subsequent IFN- $\gamma$ secretion by a subset of $\mathrm{T}$ cells. The $\mathrm{T}$ cells that secrete IFN- $\gamma$ further suppress upregulation of programmed cell death-1 (PD-1)-receptor and its ligand PD-L1 on CD11b+subset of T cells (Gaddis et al., 2013; Groeger et al., 2017). Interestingly, studies recently have demonstrated elevated PD-L1 levels in saliva from patients with oral cancers or salivary gland carcinoma (Aziz et al., 2015; Goncalves et al., 2015, 2017). Salivaderived samples have been studied as a source of biomarkers for periodontitis (Baltacioglu et al., 2014; Recker et al., 2015).

Exosomes are small $(30-100 \mathrm{~nm})$ membrane-encapsulated vesicles containing nuclei acids and protein cargo, and secreted by eukaryotic cells into the circulation (Tkach and Thery, 2016). The contents of disease cell-derived exosomes may potentially serve as a source of disease biomarkers. Salivary exosomal contents have recently been investigated for diagnosis and prognosis of a wide range of diseases (Michael et al., 2010; Lau et al., 2013; Byun et al., 2015; Machida et al., 2015; Sivadasan et al., 2015; Kim et al., 2017; Zheng et al., 2017; Han et al., 2018). Although detection of $P D-L 1 \mathrm{mRNA}$ has been reported in periodontitis (Yuan et al., 2015; Zhang et al., 2016), it is unknown whether PD-L1 mRNA can be detected in exosomes of saliva of periodontitis patients and whether the level of salivary exosomal $P D-L 1$ mRNA reflects disease status. We therefore focused on exosomes and saliva, and set to establish a protocol for isolation and detection of exosomes, and exosomal RNA in the saliva of periodontitis patients, and to characterize salivary exosomal PD-L1 as a potential marker for periodontitis.

\section{MATERIALS AND METHODS}

\section{Study Population}

This study is a prospective observational investigation at the First Affiliated Hospital of Shantou University. From June, 2017 to June, 2018, 61 periodontitis patients and 30 control subjects were enrolled in the study with informed consent. Each recruited subject was inquired in detail about the medical history and accepted a thorough periodontal examination. Diagnosis of periodontitis was based on assessment of probing pocket depth (PD) and clinical attachment loss (CAL). Patients who fulfilled the requirement of generalized chronic periodontitis according to the classification by the American Academy of Periodontology in 2007 (Slots, 2013) were recruited to the periodontitis group after periodontal examination. The non-periodontitis control group was composed of subjects with no evidence of periodontitis after examination according to the above classification. Smoking and alcohol using status was recorded for all individuals. Exclusion criteria were age $<18$ years, inability to give informed consent, congenital malformation, chronic diseases (e.g., lip cancer, gingival cancer, carcinoma of the tongue, soft palate carcinoma, jaw bone cancers, cancers of the mouth floor carcinoma, oropharyngeal cancer, salivary gland carcinoma, maxillary sinus carcinoma, cancer occurs in facial ministry skin mucous membrane, epilepsy, cardiac disease, lung disease, renal disease, positive test for human immunodeficiency virus etc.), and history of systemic antibiotic treatment or dental prophylaxis in the previous 6 months. Written informed consents were obtained from all participants in accordance with the principles established by the Helsinki Declaration. This study was approved by the Institutional Review Board of Shantou University Medical College (SUMC) (Shantou, China) under IRB protocol number: \#04-070.

\section{Clinical Evaluation}

For each individual, PD, CAL and bleeding on probing (BP) values were measured with a periodontal probe. A single calibrated examiner assessed these clinical parameters. Control individuals (control group) did not present any sign or symptom compatible with periodontal disease ( $\mathrm{PD}<3 \mathrm{~mm}$, CAL $<3 \mathrm{~mm}$ and no radiographic evidence of alveolar bone breakdown). On the other hand, the periodontitis group consisted of patients with mild, moderate or severe periodontitis (based on the Classification of Periodontal Diseases and Conditions of Armitage, 2007) (Slots, 2013) with at least one single-rooted tooth with a CAL $\geq 6 \mathrm{~mm}$ and probing depth $\geq 5 \mathrm{~mm}$.

\section{Gingival Biopsies}

Human gingivae were obtained from all periodontitis patients. Gingiva was harvested during tooth extractions for periodontal reasons in the Department of Stomatology of First Affiliated Hospital of Shantou University. Written informed consent and approval of the Ethics Committee of the SUMC were obtained.

\section{Human Saliva Collection}

Saliva was collected in the morning ( 8 am to $10 \mathrm{am}$ ) from all subjects. During the collection period, subjects were instructed to refrain from eating, drinking or using oral hygiene products for at least $1 \mathrm{~h}$ prior to collection, and received no stimulation of salivation. After rinsing the mouth with water, each subject spat $3-5 \mathrm{~mL}$ saliva into a $35-\mathrm{mm}$ dish. Subjects were reminded not to cough up mucus, and saliva was collected within $30 \mathrm{~min}$ from spitting. These saliva samples were pipetted into $1.5-\mathrm{mL}$ tubes and were kept on ice during processing which did not exceed 
$60 \mathrm{~min}$. The samples were then centrifuged at 3,000 $\times g$ for $15 \mathrm{~min}$ at $4^{\circ} \mathrm{C}$ to remove cells and cellular fragments. As much supernatant as possible was collected into new $1.5-\mathrm{mL}$ tubes and stored at $-80^{\circ} \mathrm{C}$.

\section{Gene Set Enrichment Analysis (GSEA)}

Gene set enrichment analysis $($ GSEA; v2.09 $)$ was performed to examine the association between gene sets and gene expression (Dong et al., 2017). Periodontitis gene expression profiles from an independent datasets (GSE16134) were collected from NCBI Gene Expression Omnibus (GEO) at http://www.ncbi.nlm. nih.gov/geo/. The expression levels of affected gingival tissue $(n=241)$ vs. unaffected gingival tissue $(n=69)$ from GSE16134 was ordered from high to low. We performed GSEA analysis to examine the correlation between periodontitis and the PDL1 signature pathway, followed by the protocol available at the GSEA website ${ }^{1}$.

\section{Isolation of Salivary Exosomes}

ExoQuick $^{\mathrm{TM}}$ exosomes precipitation solution was used for exosomes isolation according to the manufacturer's instructions (System Biosciences, Mountain View, CA, United States). Briefly, ExoQuick-TC ${ }^{\mathrm{TM}}$ solution was added to saliva at a 63/250 ratio and mixed by inverting the tubes several times. Exosomes were precipitated by refrigeration at $4^{\circ} \mathrm{C}$ overnight, and then collected by centrifugation twice at $1,500 \times g$ for 30 and $5 \mathrm{~min}$, respectively, in order to remove the supernatant. Supernatant was discarded, and the pellet resuspended in $300 \mu \mathrm{L}$ TRIZOL for RNA isolation or $20 \mu \mathrm{L}$ PBS for protein isolation.

\section{RT-qPCR}

Total RNA was extracted from exosomes and cell lysates using TRIzol reagent as per the manufacturer's protocol (ZYMO RESEARCH). RNA was eluted with $25 \mu \mathrm{L}$ of RNAsefree water. RT-qPCR was performed using an Absolute Blue QPCR SYBR Green Low ROX mix (Thermo Scientific) on an Applied Biosystems' 7500 real-time PCR system. The Rn value (normalized reporter value) was the fluorescent signal from SYBR Green normalized to the signal of the passive reference dye for a given reaction. No-template and no-RT reactions were performed as negative controls. All assays were performed in 3 separate RTs followed by triplicate qPCR, and the results are shown as the average fold change relative to GAPDH which served as an internal control. Primers for RT-qPCR were:

GAPDH F: 5'-TGCACCACCAACTGCTTAGC-3' GAPDH R: 5'-GGCATGGACTGTGGTCATGAG-3' PD-L1 F: 5'-TGCCGACTACAAGCGAATTACTG - $3^{\prime}$ PD-L1 R: 5'-CTGCTTGTCCAGATGACTTCGG-3'.

\section{Protein Isolation and Immunoblotting}

Exosomal pellets were resuspended in PBS and re-pelleted by centrifugation and then extracted with RIPA buffer (Santa Cruz Biotechnology). Total protein lysates were prepared and

\footnotetext{
${ }^{1}$ http://www.broadinstitute.org/gsea/
}

analyzed by immunoblotting using anti-ALIX (Cat. No. 2171; Cell Signaling Technology, Beverly, MA, United States), antiTSG101 (Cat. ab133586; Abcam, Cambridge, United Kingdom), anti-CD63 (Cat. sc-15363; Santa Cruz Biotechnology, CA, United States), anti-CD9 (Cat. sc-9148; Santa Cruz Biotechnology, CA, United States), anti-CD81 (Cat. ab109201; Abcam, Cambridge, United Kingdom), anti-Calnexin (Cat. No. 2433; Cell Signaling Technology, Beverly, MA, United States), anti-LC3 (Cat. No. 2775; Cell Signaling Technology, Beverly, MA, United States), anti-NLRP3 (Cat.19771-1-AP; Proteintech, United States) and anti-NLRP4 (Cat. NB100-56156; Novus Biologicals, United States) as described previously (Gan et al., 2016; Dong et al., 2017).

\section{Transmission Electron Microscopy}

Following exosomes isolation, the pellet was washed in PBS, and then subjected to ultracentrifugation at $120,000 \times g$ for $70 \mathrm{~min}$ to re-pellet the exosomes. The exosomes pellets were resuspended in $30 \mu \mathrm{L}$ PBS, and a $10 \mu \mathrm{L}$ aliquot of the suspension loaded onto formvar carbon-coated grids and allow to stand for $5 \mathrm{~min}$ at room temperature. Next, the exosomes were fixed in $2 \%$ paraformaldehyde for $5 \mathrm{~min}$ at room temperature and washed thrice with PBS. Excess liquid was drained by gently touching the edge of the grid with clean filter paper. The grid was slightly touched onto a drop of $2 \%$ uranyl acetate for $1 \mathrm{~min}$ and embedded in a mixture of uranyl acetate $(0.8 \%)$ and methyl cellulose $(0.13 \%)$. Excess liquid was drained off, and then the grid was allowed to air dry for several minutes prior to examination under a transmission electron microscope (JEM-1400, Hitachi, Shiga, Japan) (Raposo and Stoorvogel, 2013).

\section{Nanoparticle Tracking Analysis}

Total particles in human salivary samples were analyzed by nanoparticle tracking by the A \& P Instrument Co., Ltd. (Guangzhou, China), using a NanoSight LM10 system (NanoSight Ltd., Amesbury, United Kingdom). Each sample was diluted in nanoparticle-free PBS and analyzed three times. Data was collected and analyzed using the nanoparticle tracking analysis (NTA) software (RRID: SCR_014239, version 2.3). All measurements were recorded at room temperature.

\section{Cell Culture}

All cells were cultured in a sterile incubator maintained at $37^{\circ} \mathrm{C}$ with $5 \%$ CO2. ESCC cells and THP-1 cells were cultured in Dulbecco's modified Eagle's medium (Gibco, Paisley, United Kingdom) or RPMI-1640 medium (Gibco, Paisley, United Kingdom) supplemented with $10 \%$ heat-inactivated fetal bovine serum (Gibco, Paisley, United Kingdom), $10 \mathrm{mmol} / \mathrm{L}$ glutamine, 100 units $/ \mathrm{ml}$ penicillin (Sigma, St. Louis, MO, United States), and $100 \mu \mathrm{g} / \mathrm{ml}$ streptomycin (Sigma). TE1 cells were provided by Dr. X.C. Xu (The University of Texas MD Anderson Cancer Center, Houston, TX, United States). For LC3 detection, TE1 cells were collected after starvation (i.e., without fetal bovine serum) for $6 \mathrm{~h}$. 


\section{Statistical Analysis}

All statistical analyses were performed using the SPSS 19.0 statistical software package (SPSS Inc., Chicago, IL, United States) and Prism V6.01 (GraphPad). Summary statistics reporting means and standard errors were stated as appropriate. Statistical methods used included $t$-test, Pearson correlation and chisquare test.

\section{RESULTS}

\section{Bioinformatic Analysis of the Correlation Between Periodontitis and PD-L1}

Given the association of periodontitis with inflammation, and the importance of PD-L1 in inflammatory immunity, we analyzed database to test whether periodontitis correlates with PD-L1. GSEA analysis revealed that periodontitis is positively associated with an inflammation signature, and PD-L1 is positively correlated with an inflammation signature in periodontitis (Figure 1A). Analysis of the GEO database (GSE16134) indicated a statistically significant elevation of PD-L1 level in periodontitis patients versus control subjects (Figure 1B). These data support the hypothesis that PD-L1 is positively relevant to periodontitis.

\section{Patient Demographics and Clinical Parameters}

This pilot study was conducted at Shantou University Medical College. Sixty-one periodontitis patients and 30 control subjects were enrolled in the study (Figure 2). With an average age of 51, 29 male and 32 female patients from the First Affiliated
Hospital of Shantou University were recruited. There are also 30 control subjects (14 male and 16 female) were enrolled in this study with an average age of 52. Among demographic and clinicopathological characteristics, age, gender, tobacco use, alcohol use, hypertension and diabetes did not show any significant differences between the two groups (Table 1).

\section{Extraction and Characterization of Exosomes From Human Saliva}

Isolation of exosomes from human saliva was confirmed by transmission electron microscopy (TEM) (i.e., spherical membrane-bound particles with diameters between 30 and $100 \mathrm{~nm}$ ) (Thery et al., 2009; Raposo and Stoorvogel, 2013; Figure 3A) and nanoparticle tracking analysis showing that human exosomes had an average diameter of $95 \mathrm{~nm}$ (Filipe et al., 2010; Webber and Clayton, 2013; Figure 3B). Immunoblotting of exosomal markers (ALIX, TSG101, CD63, CD9, CD81) and the intracellular protein that is not present in exosomes (Calnexin) (Mathivanan and Simpson, 2009; Taylor and GercelTaylor, 2011; Lotvall et al., 2014; Figure 3C) were performed for further confirmation. For eliminating the possibility of contamination by autophagosomes where significant amount of PD-L1 would be found, LC3 II, the marker of autophagosome was evaluated by immunoblotting; salivary exosomes samples did not contain LC3 while lysate of TE1 cells cultured in DMEM without serum for $6 \mathrm{~h}$ (positive control) showed the presence of LC3 II (Figure 3C). In addition, the marker of endoplasmic reticulum, calnexin, and the markers of inflammasomes were also analyzed by immunoblotting to exclude contamination of exosomes. The immunoblotting showed that calnexin, NLRP3

\section{A}

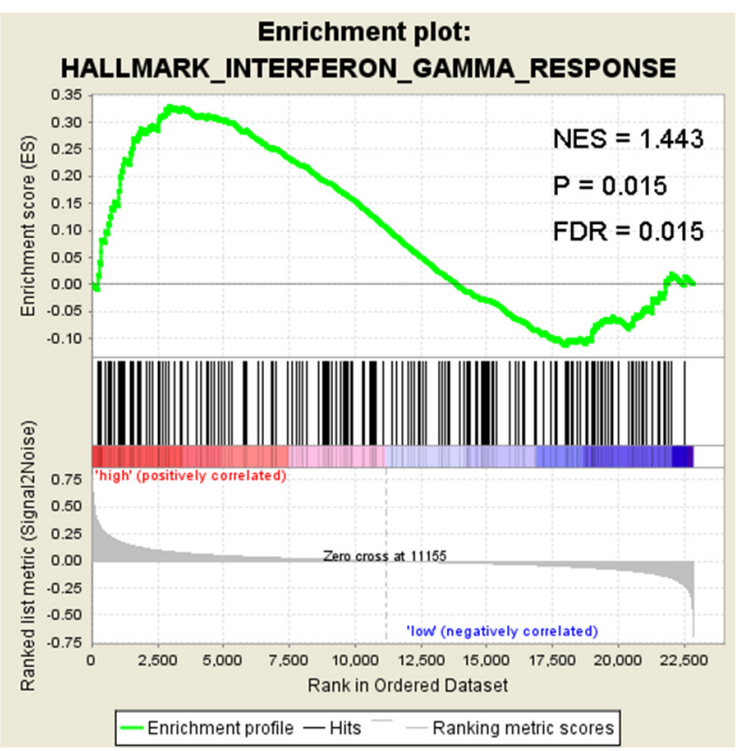

B

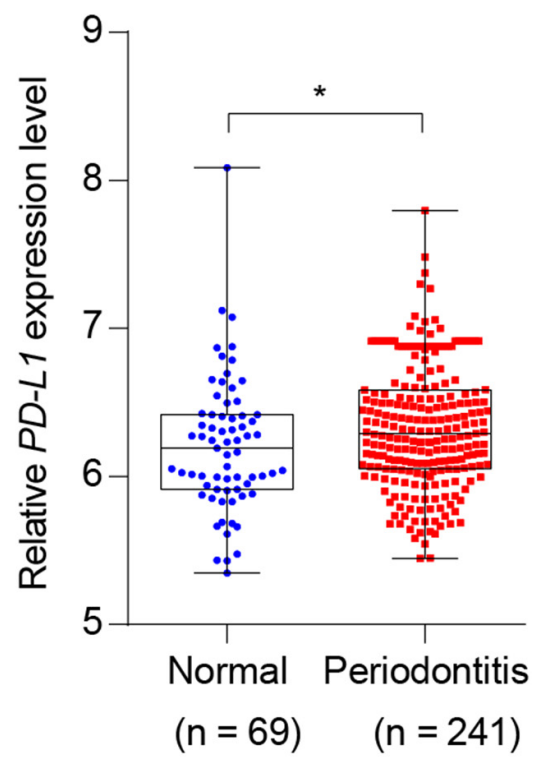

FIGURE 1 | Periodontitis correlated with inflammation and PD-L1 signatures. (A) PD-L1 gene signatures were analyzed by GSEA using the gene sets (GSE 16134) derived from periodontitis patients and normal subjects. FDR $=0.015$. (B) Quantified results show the expression level of $P D-L 1$ in the gene sets (GSE 16134). Periodontitis patients $(n=241)$ compared with normal subjects $(n=69)$. Error bars indicate SEM. $* P<0.05$ by Student's $t$-test. 


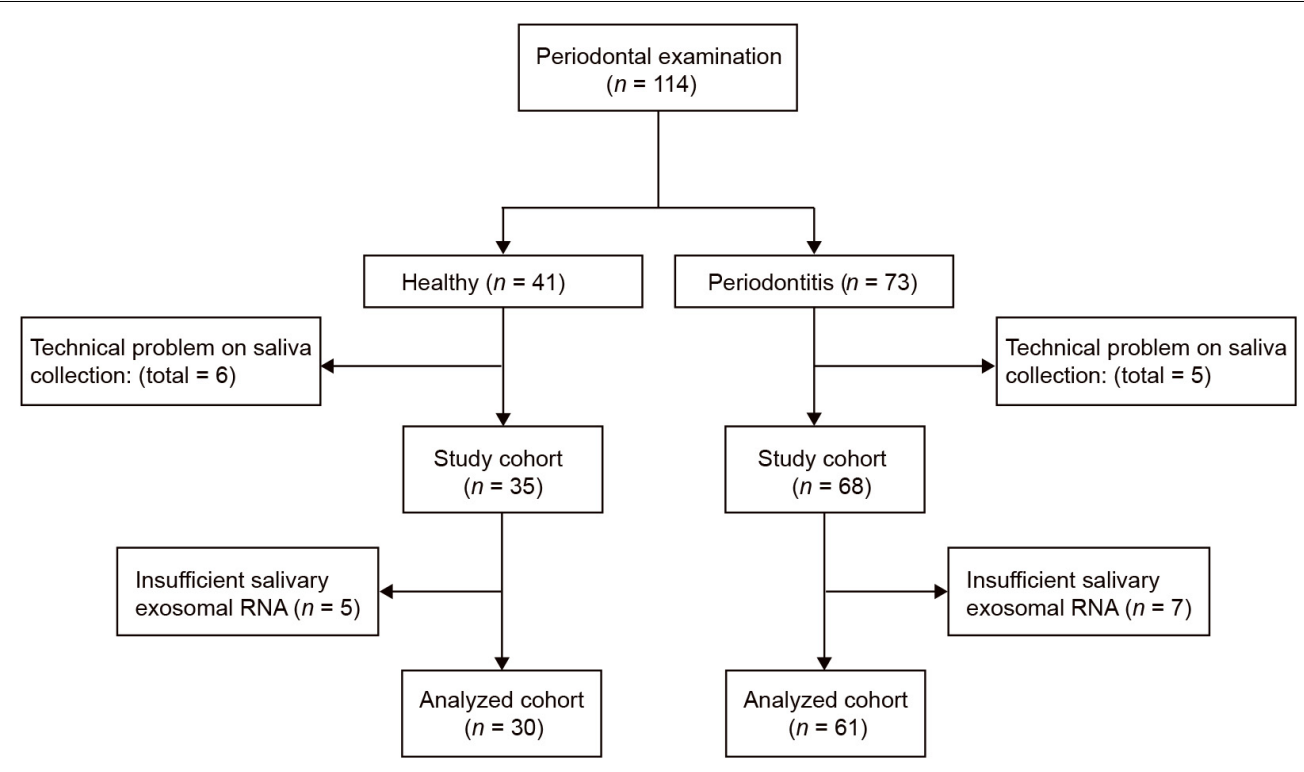

FIGURE 2 | Flow diagram of periodontitis patients and control subjects. Participant numbers are accounted for by the flow diagram for the study.

TABLE 1 | The demographics and clinicopathological characteristics of the periodontitis patients and control subjects.

\begin{tabular}{|c|c|c|c|}
\hline Variables & Control $(n=30) n(\%)$ & Patient $(n=61) n(\%)$ & $P$-value* \\
\hline \multicolumn{4}{|l|}{ Age (years) } \\
\hline$\leq 50$ & 18 (31.6) & $39(68.4)$ & 0.715 \\
\hline$>50$ & $12(35.3)$ & $22(64.7)$ & \\
\hline \multicolumn{4}{|l|}{ Gender } \\
\hline Female & $21(39.6)$ & $32(60.4)$ & 0.111 \\
\hline Male & $9(23.7)$ & 29 (76.3) & \\
\hline \multicolumn{4}{|l|}{ Tobacco use } \\
\hline No & $19(32.2)$ & $40(67.8)$ & 0.833 \\
\hline Yes & $11(34.4)$ & $21(65.6)$ & \\
\hline \multicolumn{4}{|l|}{ Alcohol use } \\
\hline No & $20(30.8)$ & $45(69.2)$ & 0.481 \\
\hline Yes & $10(48.5)$ & $16(61.5)$ & \\
\hline \multicolumn{4}{|c|}{ Hypertension } \\
\hline No & $23(35.9)$ & $41(64.1)$ & 0.353 \\
\hline Yes & 7 (25.9) & $20(74.1)$ & \\
\hline \multicolumn{4}{|l|}{ Diabetes } \\
\hline No & $27(36.5)$ & $47(63.5)$ & 0.136 \\
\hline Yes & $3(17.6)$ & $14(82.4)$ & \\
\hline \multicolumn{4}{|l|}{ Stage } \\
\hline Mild & NA & 26 & \\
\hline Moderate & NA & 21 & \\
\hline Severe & NA & 14 & \\
\hline
\end{tabular}

*P-values were calculated by $\chi^{2}$ test.

and NLRP4 could be detected in positive control but not in salivary exosomes samples (Figure 3C). Thus, exosomes were successfully isolated from saliva of periodontitis patients without significant contamination by autophagosomes, endoplasmic reticulum or inflammasomes.

\section{Salivary Exosomal PD-L1 mRNA Was Elevated in Periodontitis Patients}

We next determined the salivary exosomal and gingival PD-L1 mRNA expression, in 61 periodontitis patients and 30 control subjects, by qPCR analysis. As shown in Figure 4A, 45 of 61 cases $(73.8 \%)$ had increased salivary exosomal $P D-L 1$ expression compared with the mean salivary exosomal $P D-L 1$ expression in control subjects. Mean salivary exosomal $P D-L 1$ expression in the periodontitis patients was found to be about 10 -fold higher when compared with the paired control subjects $(P<0.001)$. Similarly, mean gingival $P D-L 1$ mRNA expression in the periodontitis patients was more than 10 -fold higher than that in control subjects (Figure 4B, $P<0.001$ ). More importantly, salivary exosomal $P D-L 1$ mRNA levels highly correlated with gingival $P D$-L1 mRNA levels in periodontitis patients $(r=0.800$ and $P<0.001$, Pearson's correlation test; Figure 4B). Collectively, our results strongly suggested that salivary exosomal PD-L1 mRNA could be a feasible biomarker of periodontitis.

\section{Clinical Relevance of Salivary Exosomal PD-L1 mRNA in Periodontitis Patients}

We further assessed the association between salivary exosomal $P D-L 1$ mRNA and clinical parameters in 61 periodontitis patients. The median value (i.e., relative value of salivary exosomal $P D-L 1$ mRNA $=9.90)$ was chosen to classify patients into high- $(n=31)$ and low-PD-L1 $(n=30)$ groups (Table 2$)$. High $P D-L 1$ expression was only associated with advanced stage $\left(P=0.005 ; \chi^{2}\right.$ test; Table 2$)$, and $P D-L 1$ expression was not found to be statistically significantly associated with other clinicopathological parameters (Table 2). Our results demonstrated that salivary exosomal $P D-L 1$ mRNA could reflect the stage of periodontitis, suggesting that PD-L1 may be relevant to the disease progression. 
A

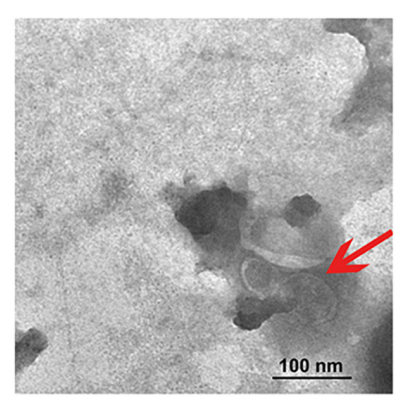

C
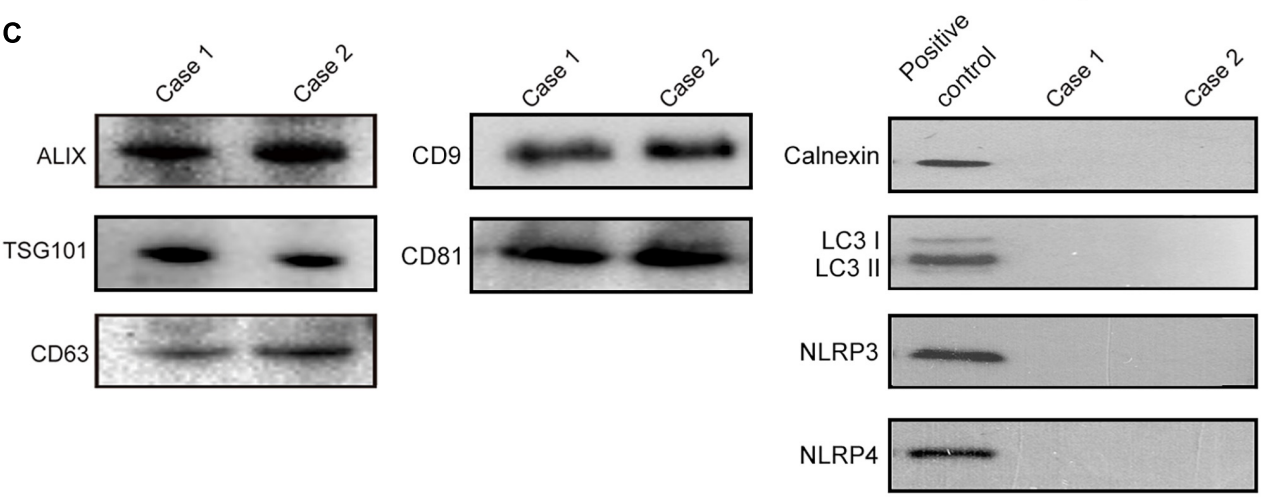

B

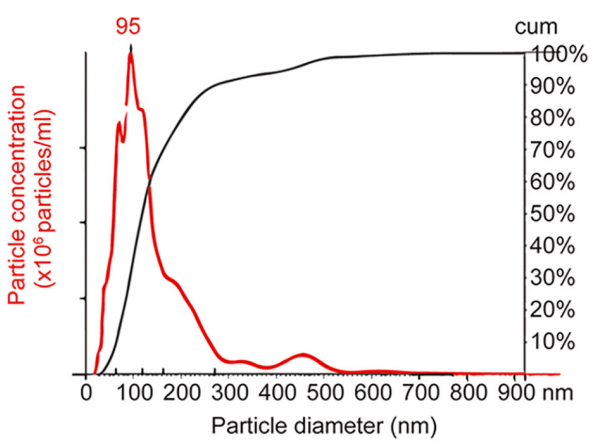

FIGURE 3 | Identification of salivary exosomes in periodontitis patients and normal subjects. (A) Transmission electron microscopy of exosomes isolated from human saliva. Scale bar: $100 \mathrm{~nm}$. (B) Exosomes concentration and size distribution by NanoSight analysis of human saliva. (C) Immunoblotting showed the exosomal membrane markers (ALIX, TSG101 CD63, CD9 and CD81), the intracellular protein Calnexin, the marker of autophagosome LC3 and markers of inflammasome (NLRP3 and NLRP4) in exosomes isolated from the saliva of one normal subject (case 01) and one periodontitis patient (case 02). Positive control for Calnexin was TE1 cells, and positive control for LC3 was TE1 cells after starvation for $6 \mathrm{~h}$. Positive control for NLRP3 and NLRP4 was THP-1 cells.

\section{A}

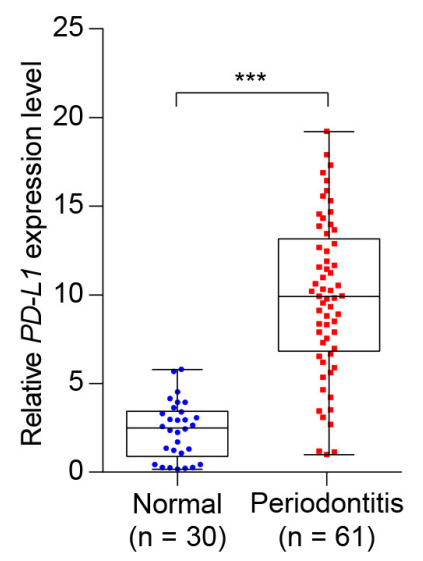

B

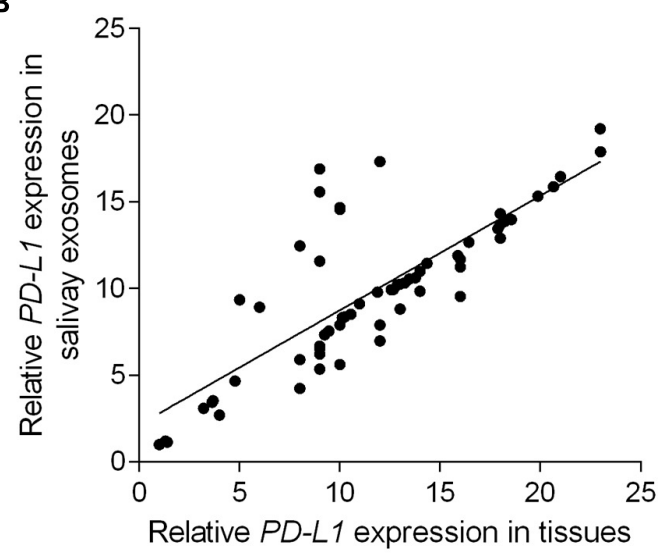

FIGURE 4 | Detection of salivary exosomal $P D-L 1$ in periodontitis patients and normal subjects. (A) Salivary exosomal PD- $L 1$ was measured by RT-qPCR in periodontitis patients $(n=61)$ and normal controls $(n=30)$. Error bars indicate SEM. ${ }^{* * *} P<0.001$ by Student's $t$-test. (B) Salivary exosomal $P D-L 1$ correlated with periodontal tissue $P D-L 1$ expression in periodontitis patients (Pearson's correlation test).

\section{DISCUSSION}

We characterized PD-L1 mRNA expression in exosomes derived from saliva of periodontitis patients, and have evaluated the clinical relevance of the levels of salivary exosomes $P D-L 1$ mRNA in the disease. One of the main findings was that the level of salivary exosomes $P D-L 1$ mRNA in periodontitis patients is highly distinct relative to non-periodontitis controls. Moreover, high level of salivary exosomes $P D-L 1$ was associated with an advanced stage of periodontitis, suggesting it can reflect disease progression. This is the first to establish a procedure of detection of saliva-based exosomal $P D-L 1$ in disease, and 
TABLE 2 | The clinicopathological characteristics related to $P D-L 1$ expression in periodontitis patients.

\begin{tabular}{|c|c|c|c|c|}
\hline \multirow[t]{2}{*}{ Variables } & \multirow[t]{2}{*}{ No. of patients } & \multicolumn{2}{|c|}{$P D-L 1$ expression } & \multirow[t]{2}{*}{$P$-value* } \\
\hline & & Low, $n(\%)$ & High, $n(\%)$ & \\
\hline Total samples & 61 & $30(49.2)$ & $31(50.8)$ & \\
\hline \multicolumn{5}{|l|}{ Age (years) } \\
\hline$\geq 50$ & 39 & $18(46.2)$ & $21(53.8)$ & 0.529 \\
\hline$<50$ & 22 & $12(54.5)$ & $10(45.5)$ & \\
\hline \multicolumn{5}{|l|}{ Gender } \\
\hline Female & 32 & $19(59.4)$ & $13(40.6)$ & 0.653 \\
\hline Male & 29 & $11(37.9)$ & $18(62.1)$ & \\
\hline \multicolumn{5}{|l|}{ Tobacco use } \\
\hline No & 40 & $21(52.5)$ & 19 (47.5) & 0.474 \\
\hline Yes & 21 & $9(42.9)$ & $12(57.1)$ & \\
\hline \multicolumn{5}{|l|}{ Alcohol use } \\
\hline No & 45 & $25(55.6)$ & $20(44.4)$ & 0.095 \\
\hline Yes & 16 & $5(31.3)$ & $11(68.8)$ & \\
\hline \multicolumn{5}{|l|}{ Hypertension } \\
\hline No & 41 & $22(53.7)$ & 19(46.3) & 0.316 \\
\hline Yes & 20 & $8(40.0)$ & $12(60.0)$ & \\
\hline \multicolumn{5}{|l|}{ Diabetes } \\
\hline No & 47 & $25(53.2)$ & $22(46.8)$ & 0.251 \\
\hline Yes & 14 & $5(35.7)$ & $9(64.3)$ & \\
\hline \multicolumn{5}{|l|}{ Stage } \\
\hline Mild & 26 & $19(73.1)$ & $7(26.9)$ & 0.005 \\
\hline Moderate & 21 & 7 (33.3) & $14(66.7)$ & \\
\hline Severe & 14 & 4 (28.6) & $10(71.4)$ & \\
\hline
\end{tabular}

*P-values were calculated by $x^{2}$ test. High in this analysis is based on a PD-L1 level > 9.9 (median); the remaining individuals were classified as low.

the first salivary exosomal biomarker for periodontitis. In the majority of previous investigations of periodontitis biomarker, the specimens are either gingival tissues or gingival crevicular fluids (GCF) (Buduneli and Kinane, 2011; Ebersole et al., 2014; Jagannathan et al., 2014; Kebschull et al., 2014). However, sample collection and procedures for both gingival tissues and GCF are challenging: gingival tissue biopsy involves acquisition from invasive and limited tissue, and GCF sample collection involves sampling of a minute amount of fluid on filter paper strips, which requires a longer sampling time. Saliva-based assay overcomes these forgoing problems, as shown by our current study which involves an easy, non-invasive, and rapid collection of salivary specimens. We also demonstrate that mRNA can be extracted well from salivary exosomes, supporting the notion that exosomes-derived samples prevent mRNA from degradation (Kim et al., 2005; Soung et al., 2017). To our knowledge, this is the first to detect $P D-L 1$ in exosomes and in saliva in periodontitis. The procedure described in the current study may be used for detecting mRNAs in oral diseases as well as in other systemic diseases.

PD-L1 has been reported to play an important role in a wide range of cancers and inflammation-originated diseases including periodontitis (Ota et al., 2015; Clark et al., 2016; Concha-Benavente et al., 2016; Zhang et al., 2016; Groeger et al., 2017). The role of PD-L1 to inhibit destruction of inflammatory tissues has been reported (Scandiuzzi et al., 2014). In two previous reports, it has been shown that PDL1 expression in periodontitis tissues is increased in mild and moderate periodontitis (Yuan et al., 2015; Zhang et al., 2016). However, it is unclear whether PD-L1 expression correlates with disease status of chronic periodontitis. It has been also reported that expression of $\mathrm{PD}-1$ and $\mathrm{PD}-\mathrm{L} 1$ in peripheral $\mathrm{CD}^{+} \mathrm{T}$ lymphocytes and $\mathrm{CD}^{+} \mathrm{T}$ lymphocyte of chronic periodontitis patients was upregulated (Zhu et al., 2014). High expression of PD-L1 in host cells may contribute to the chronicity of inflammatory disorders (Zamani et al., 2016). The previous work showed that the PD-L1 expression in different compartments may be different and have different roles in inflammatory disease. Therefore, further correlation between PD-L1 and periodontitis are worthy to investigated. In our current study, it suggests that assay of exosomebased PD-L1 mRNA in saliva has potential to distinguish periodontitis from the healthy, and the levels correlate with the severity/stage of periodontitis. The increased expression of PDL1 in severe periodontitis might due to the requirement of the body for more PD-L1 to inhibit destruction of inflammatory tissues. It has been shown that tumor cells upregulate PDL1 expression to evade host immune response and thereby to maintain disease (Flies et al., 2016; Wang et al., 2016; Lakin et al., 2017). Moreover, the potential for PD-L1 to serve as a disease biomarker has also been revealed (Colwell, 2015; Fusi et al., 2015). PD-L1 is upregulated in cancers, such as glioblastoma (Wang et al., 2016), ovarian cancer (Abiko et al., 2013), lung cancer and oral carcinoma (Kim et al., 2005; Ota et al., 2015), and upregulated PD-L1 predicts worse survival. In periodontitis, $\mathrm{PD}-\mathrm{L} 1$ has been detected in gingival tissues, gingival crevicular fluid (GCF) and blood (Olsen et al., 2016; Zhang et al., 2016; Groeger et al., 2017). Our analysis of published database validated that periodontitis specimens harbor high levels of PD-L1. Although detection of PD-L1 has been widely reported in tissues and blood (Fusi et al., 2015; Wang et al., 2016; Groeger et al., 2017), there are no publications for PD-L1 detection in salivary exosomes. We show that PD-L1 is present in exosomes of saliva from periodontitis patients, suggesting the possibility that PD-L1 is enriched in exosomes. Our results are consistent with a recent study that exosomes released from melanoma cells were found to carry a remarkable amount of PD-L1 on their surfaces (Chen et al., 2018). We here described a more convenient procedure for isolation and detection of PD-L1 in salivary exosomes. Since PD-L1 involves many diseases including cancers (i.e., oral cancers) and immune diseases (Gianchecchi et al., 2013; Dai et al., 2014; Roemer et al., 2016), the protocol established in current study may provide a platform for easy and rapid assay of PD-L1 in variety of applications. The limitation of the study was the relatively small size of the patient cohorts. Further investigations using larger and multiple cohorts are worthy to be performed to validate the findings of the current studies.

In summary, we described the procedure for isolating mRNA from exosomes derived from saliva of periodontitis patients. Our further assay showed that exosomal $P D-L 1$ in saliva was 
enriched in periodontitis and was associated with advanced stage of disease. These findings are worthy to validate in further investigations with expanding samples.

\section{AUTHOR CONTRIBUTIONS}

HZ conceived and designed the experiments. JY and DY provided patients and the study materials. YL, KL, XX, ZY, $\mathrm{HD}$, and $\mathrm{ZJ}$ performed the in vitro experiments about patient specimen analysis, bioinformatics assay, and analyzed data. JY, YL, XX, S-CY, and HZ interpreted data and wrote the manuscript. JY, S-CY, DY, and HZ contributed to discussion and reviewed the manuscript.

\section{REFERENCES}

Abiko, K., Mandai, M., Hamanishi, J., Yoshioka, Y., Matsumura, N., Baba, T., et al. (2013). PD-L1 on tumor cells is induced in ascites and promotes peritoneal dissemination of ovarian cancer through CTL dysfunction. Clin. Cancer Res. 19, 1363-1374. doi: 10.1158/1078-0432.CCR-12-2199

Aziz, S., Ahmed, S. S., Ali, A., Khan, F. A., Zulfiqar, G., Iqbal, J., et al. (2015). Salivary immunosuppressive cytokines IL-10 and IL-13 are significantly elevated in oral squamous cell carcinoma patients. Cancer Invest. 33, 318-328. doi: 10.3109/07357907.2015.1041642

Baeza, M., Garrido, M., Hernandez-Rios, P., Dezerega, A., Garcia-Sesnich, J., Strauss, F., et al. (2016). Diagnostic accuracy for apical and chronic periodontitis biomarkers in gingival crevicular fluid: an exploratory study. J. Clin. Periodontol. 43, 34-45. doi: 10.1111/jcpe.12479

Baltacioglu, E., Yuva, P., Aydin, G., Alver, A., Kahraman, C., Karabulut, E., et al. (2014). Lipid peroxidation levels and total oxidant/antioxidant status in serum and saliva from patients with chronic and aggressive periodontitis. oxidative stress index: a new biomarker for periodontal disease? J. Periodontol. 85, 1432-1441. doi: 10.1902/jop.2014.130654

Buduneli, N., and Kinane, D. F. (2011). Host-derived diagnostic markers related to soft tissue destruction and bone degradation in periodontitis. J. Clin. Periodontol. 38(Suppl. 11), 85-105. doi: 10.1111/j.1600-051X.2010.01670.x

Byun, J. S., Hong, S. H., Choi, J. K., Jung, J. K., and Lee, H. J. (2015). Diagnostic profiling of salivary exosomal microRNAs in oral lichen planus patients. Oral Dis. 21, 987-993. doi: 10.1111/odi.12374

Chen, G., Huang, A. C., Zhang, W., Zhang, G., Wu, M., Xu, W., et al. (2018). Exosomal PD-L1 contributes to immunosuppression and is associated with anti-PD-1 response. Nature 560, 382-386. doi: 10.1038/s41586-018-0392-8

Clark, C. A., Gupta, H. B., Sareddy, G., Pandeswara, S., Lao, S., Yuan, B., et al. (2016). Tumor-Intrinsic PD-L1 signals regulate cell growth, pathogenesis, and autophagy in ovarian cancer and melanoma. Cancer Res. 76, 6964-6974. doi: 10.1158/0008-5472.CAN-16-0258

Colwell, J. (2015). Is PD-L1 expression a biomarker of response? Cancer Discov. 5:1232. doi: 10.1158/2159-8290.CD-ND2015-004

Concha-Benavente, F., Srivastava, R. M., Trivedi, S., Lei, Y., Chandran, U., Seethala, R. R., et al. (2016). Identification of the cell-intrinsic and -extrinsic pathways downstream of EGFR and IFNgamma that induce PD-L1 expression in head and neck cancer. Cancer Res. 76, 1031-1043. doi: 10.1158/0008-5472.CAN-152001

Dai, S., Jia, R., Zhang, X., Fang, Q., and Huang, L. (2014). The PD-1/PD-Ls pathway and autoimmune diseases. Cell Immunol. 290, 72-79. doi: 10.1016/j.cellimm. 2014.05.006

Dong, H., Ma, L., Gan, J., Lin, W., Chen, C., Yao, Z., et al. (2017). PTPRO represses ERBB2-driven breast oncogenesis by dephosphorylation and endosomal internalization of ERBB2. Oncogene 36, 410-422. doi: 10.1038/onc.2016.213

Ebersole, J. L., Kirakodu, S., Novak, M. J., Stromberg, A. J., Shen, S., Orraca, L., et al. (2014). Cytokine gene expression profiles during initiation, progression and resolution of periodontitis. J. Clin. Periodontol. 41, 853-861. doi: 10.1111/ jcpe. 12286

\section{FUNDING}

This work was supported by the following funding agencies: National Natural Science Foundation of China (81572876, 81773087, 81071736, and 30973508) to HZ; Li Ka Shing Foundation Grant for Joint Research Program between Shantou University and Technion-Israel Institute of Technology (43209504) to HZ, Shantou University, Guangdong Province.

\section{ACKNOWLEDGMENTS}

We thank the dentists and patients who participated in these studies.

Filipe, V., Hawe, A., and Jiskoot, W. (2010). Critical evaluation of nanoparticle tracking analysis (NTA) by nanosight for the measurement of nanoparticles and protein aggregates. Pharm. Res. 27, 796-810. doi: 10.1007/s11095-0100073-2

Flies, A. S., Lyons, A. B., Corcoran, L. M., Papenfuss, A. T., Murphy, J. M., Knowles, G. W., et al. (2016). PD-L1 Is not constitutively expressed on tasmanian devil facial tumor cells but is strongly upregulated in response to IFN-gamma and can be expressed in the tumor microenvironment. Front. Immunol. 7:581. doi: 10.3389/fimmu.2016.00581

Fusi, A., Festino, L., Botti, G., Masucci, G., Melero, I., Lorigan, P., et al. (2015). PDL1 expression as a potential predictive biomarker. Lancet Oncol. 16, 1285-1287. doi: 10.1016/S1470-2045(15)00307-1

Gaddis, D. E., Maynard, C. L., Weaver, C. T., Michalek, S. M., and Katz, J. (2013). Role of TLR2-dependent IL-10 production in the inhibition of the initial IFNgamma T cell response to Porphyromonas gingivalis. J. Leukoc. Biol. 93, 21-31. doi: $10.1189 /$ jlb.0512220

Gan, J., Ke, X., Jiang, J., Dong, H., Yao, Z., Lin, Y., et al. (2016). Growth hormonereleasing hormone receptor antagonists inhibit human gastric cancer through downregulation of PAK1-STAT3/NF-kappaB signaling. Proc. Natl. Acad. Sci. U.S.A. 113, 14745-14750. doi: 10.1073/pnas.1618582114

Gianchecchi, E., Delfino, D. V., and Fierabracci, A. (2013). Recent insights into the role of the PD-1/PD-L1 pathway in immunological tolerance and autoimmunity. Autoimmun. Rev. 12, 1091-1100. doi: 10.1016/j.autrev.2013. 05.003

Goncalves, A. S., Arantes, D. A., Bernardes, V. F., Jaeger, F., Silva, J. M., Silva, T. A., et al. (2015). Immunosuppressive mediators of oral squamous cell carcinoma in tumour samples and saliva. Hum. Immunol. 76, 52-58. doi: 10.1016/j.humimm. 2014.11.002

Goncalves, A. S., Mosconi, C., Jaeger, F., Wastowski, I. J., Aguiar, M. C. F., Silva, T. A., et al. (2017). Overexpression of immunomodulatory mediators in oral precancerous lesions. Hum. Immunol. 78, 752-757. doi: 10.1016/j.humimm. 2017.09.003

Groeger, S., Jarzina, F., Mamat, U., and Meyle, J. (2017). Induction of B7$\mathrm{H} 1$ receptor by bacterial cells fractions of Porphyromonas gingivalis on human oral epithelial cells: B7-H1 induction by Porphyromonas gingivalis fractions. Immunobiology 222, 137-147. doi: 10.1016/j.imbio.2016. 10.011

Han, D. H., Shin, H. S., Kim, M. S., Paek, D., and Kim, H. D. (2012). Group of serum inflammatory markers and periodontitis-metabolic syndrome coexistence in Koreans. J. Periodontol. 83, 612-620. doi: 10.1902/jop.2011.110304

Han, Y., Jia, L., Zheng, Y., and Li, W. (2018). Salivary exosomes: emerging roles in systemic disease. Int. J. Biol. Sci. 14, 633-643. doi: 10.7150/ijbs.25018

Jagannathan, R., Lavu, V., and Rao, S. R. (2014). Comparison of the proportion of non-classic (CD14+CD16+) monocytes/macrophages in peripheral blood and gingiva of healthy individuals and patients with chronic periodontitis. J. Periodontol. 85, 852-858. doi: 10.1902/jop.2013.120658

Kebschull, M., Demmer, R. T., Grun, B., Guarnieri, P., Pavlidis, P., and Papapanou, P. N. (2014). Gingival tissue transcriptomes identify distinct periodontitis phenotypes. J. Dent. Res. 93, 459-468. doi: 10.1177/0022034514527288 
Kim, J., Shin, H., and Park, J. (2017). RNA in salivary extracellular vesicles as a possible tool for systemic disease diagnosis. J. Dent. Res. 96, 938-944. doi: $10.1177 / 0022034517702100$

Kim, J. W., Wieckowski, E., Taylor, D. D., Reichert, T. E., Watkins, S., and Whiteside, T. L. (2005). Fas ligand-positive membranous vesicles isolated from sera of patients with oral cancer induce apoptosis of activated T lymphocytes. Clin. Cancer Res. 11, 1010-1020.

Kimak, A., Strycharz-Dudziak, M., Bachanek, T., and Kimak, E. (2015). Lipids and lipoproteins and inflammatory markers in patients with chronic apical periodontitis. Lipids Health Dis. 14:162. doi: 10.1186/s12944-015-0156-5

Koregol, A. C., More, S. P., Nainegali, S., Kalburgi, N., and Verma, S. (2011). Analysis of inorganic ions in gingival crevicular fluid as indicators of periodontal disease activity: a clinico-biochemical study. Contemp. Clin. Dent. 2, 278-282. doi: 10.4103/0976-237X.91788

Lakin, N., Rulach, R., Nowicki, S., and Kurian, K. M. (2017). Current advances in checkpoint inhibitors: lessons from non-central nervous system cancers and potential for glioblastoma. Front. Oncol. 7:141. doi: 10.3389/fonc.2017.00141

Lau, C., Kim, Y., Chia, D., Spielmann, N., Eibl, G., Elashoff, D., et al. (2013). Role of pancreatic cancer-derived exosomes in salivary biomarker development. J. Biol. Chem. 288, 26888-26897. doi: 10.1074/jbc.M113.452458

Lotvall, J., Hill, A. F., Hochberg, F., Buzas, E. I., Di Vizio, D., Gardiner, C., et al. (2014). Minimal experimental requirements for definition of extracellular vesicles and their functions: a position statement from the international society for extracellular vesicles. J. Extracell. Vesicles 3:26913. doi: 10.3402/jev.v3.26913

Machida, T., Tomofuji, T., Ekuni, D., Maruyama, T., Yoneda, T., Kawabata, Y., et al. (2015). MicroRNAs in salivary exosome as potential biomarkers of aging. Int. J. Mol. Sci. 16, 21294-21309. doi: 10.3390/ijms160921294

Mathivanan, S., and Simpson, R. J. (2009). ExoCarta: a compendium of exosomal proteins and RNA. Proteomics 9, 4997-5000. doi: 10.1002/pmic.200900351

Michael, A., Bajracharya, S. D., Yuen, P. S., Zhou, H., Star, R. A., Illei, G. G., et al. (2010). Exosomes from human saliva as a source of microRNA biomarkers. Oral Dis. 16, 34-38. doi: 10.1111/j.1601-0825.2009.01604.x

Olsen, I., Taubman, M. A., and Singhrao, S. K. (2016). Porphyromonas gingivalis suppresses adaptive immunity in periodontitis, atherosclerosis, and Alzheimer's disease. J. Oral Microbiol. 8:33029. doi: 10.3402/jom.v8.33029

Ota, K., Azuma, K., Kawahara, A., Hattori, S., Iwama, E., Tanizaki, J., et al. (2015). Induction of PD-L1 expression by the EML4-ALK oncoprotein and downstream signaling pathways in non-small cell lung cancer. Clin. Cancer Res. 21, 4014-4021. doi: 10.1158/1078-0432.CCR-15-0016

Raposo, G., and Stoorvogel, W. (2013). Extracellular vesicles: exosomes, microvesicles, and friends. J. Cell Biol. 200, 373-383. doi: 10.1083/jcb.201211138

Recker, E. N., Brogden, K. A., Avila-Ortiz, G., Fischer, C. L., Pagan-Rivera, K., Dawson, D. V., et al. (2015). Novel biomarkers of periodontitis and/or obesity in saliva-An exploratory analysis. Arch. Oral. Biol. 60, 1503-1509. doi: 10.1016/ j.archoralbio.2015.07.006

Roemer, M. G., Advani, R. H., Ligon, A. H., Natkunam, Y., Redd, R. A., Homer, H., et al. (2016). PD-L1 and PD-L2 genetic alterations define classical hodgkin lymphoma and predict outcome. J. Clin. Oncol. 34, 2690-2697. doi: 10.1200/ JCO.2016.66.4482

Scandiuzzi, L., Ghosh, K., Hofmeyer, K. A., Abadi, Y. M., Lazar-Molnar, E., Lin, E. Y., et al. (2014). Tissue-expressed B7-H1 critically controls intestinal inflammation. Cell Rep. 6, 625-632. doi: 10.1016/j.celrep.2014.01.020
Sivadasan, P., Gupta, M. K., Sathe, G. J., Balakrishnan, L., Palit, P., Gowda, H., et al. (2015). Human salivary proteome-a resource of potential biomarkers for oral cancer. J. Proteomics 127(Pt A), 89-95. doi: 10.1016/j.jprot.2015.05.039

Slots, J. (2013). Periodontology: past, present, perspectives. Periodontol 200062 , 7-19. doi: 10.1111/prd.12011

Soung, Y. H., Ford, S., Zhang, V., and Chung, J. (2017). Exosomes in cancer diagnostics. Cancers 9:8. doi: 10.3390/cancers9010008

Taylor, D. D., and Gercel-Taylor, C. (2011). Exosomes/microvesicles: mediators of cancer-associated immunosuppressive microenvironments. Semin. Immunopathol. 33, 441-454. doi: 10.1007/s00281-010-0234-8

Thery, C., Ostrowski, M., and Segura, E. (2009). Membrane vesicles as conveyors of immune responses. Nat. Rev. Immunol. 9, 581-593. doi: 10.1038/nri 2567

Tkach, M., and Thery, C. (2016). Communication by extracellular vesicles: where we are and where we need to go. Cell 164, 1226-1232. doi: 10.1016/j.cell.2016. 01.043

Wang, Z., Zhang, C., Liu, X., Sun, L., Li, G., Liang, J., et al. (2016). Molecular and clinical characterization of PD-L1 expression at transcriptional level via 976 samples of brain glioma. Oncoimmunology 5:e1196310. doi: 10.1080/2162402X. 2016.1196310

Webber, J., and Clayton, A. (2013). How pure are your vesicles? J. Extracell. Vesicles 2:19861. doi: 10.3402/jev.v2i0.19861

Yuan, W., Wang, X., Zhang, J., Zhou, W., Feng, Y., Chen, J., et al. (2015). [Programmed death ligand 1 negatively regulates inflammatory response of chronic periodontitis]. Hua Xi Kou Qiang Yi Xue Za Zhi 33, 366-369.

Zamani, M. R., Aslani, S., Salmaninejad, A., Javan, M. R., and Rezaei, N. (2016). PD1/PD-L and autoimmunity: a growing relationship. Cell Immunol. 310, 27-41. doi: 10.1016/j.cellimm.2016.09.009

Zhang, J., Wang, C. M., Zhang, P., Wang, X., Chen, J., Yang, J., et al. (2016). Expression of programmed death 1 ligand 1 on periodontal tissue cells as a possible protective feedback mechanism against periodontal tissue destruction. Mol. Med. Rep. 13, 2423-2430. doi: 10.3892/mmr.2016.4824

Zheng, X., Chen, F., Zhang, Q., Liu, Y., You, P., Sun, S., et al. (2017). Salivary exosomal PSMA7: a promising biomarker of inflammatory bowel disease. Protein Cell 8, 686-695. doi: 10.1007/s13238-017-0413-7

Zhu, D., Liu, F., Dai, F., Luo, X., and Hong, B. (2014). Expression of programmed death-1 and programmed death ligand- 1 in the peripheral T-lymphocytes from patients with chronic periodontitis. Zhonghua Kou Qiang Yi Xue Za Zhi 49, 216-219.

Conflict of Interest Statement: The authors declare that the research was conducted in the absence of any commercial or financial relationships that could be construed as a potential conflict of interest.

Copyright (C) 2019 Yu, Lin, Xiong, Li, Yao, Dong, Jiang, Yu, Yeung and Zhang. This is an open-access article distributed under the terms of the Creative Commons Attribution License (CC BY). The use, distribution or reproduction in other forums is permitted, provided the original author(s) and the copyright owner(s) are credited and that the original publication in this journal is cited, in accordance with accepted academic practice. No use, distribution or reproduction is permitted which does not comply with these terms. 